\title{
Lesson of the month 1: Diabetic ketoacidosis in established renal failure
}

\author{
Authors: Ravi Varma ${ }^{A}$ and Mahzuz Karim ${ }^{B}$
}

\begin{abstract}
Diabetic ketoacidosis (DKA) is a common medical emergency. The pathophysiology of DKA in patients with advanced chronic kidney disease differs significantly from patients with preserved renal function. We describe a patient with pre-dialysis renal failure who presented with DKA. This case highlights the importance of tailoring the established management guidelines appropriately.
\end{abstract}

KEYWORDS: Diabetic ketoacidosis, renal failure, dialysis

\section{Case history}

A 70-year-old woman presented to the emergency department with general malaise, which had developed over a few days. She had a background of adult polycystic kidney disease and had received a renal transplant 16 years earlier. This was complicated by the development of new onset diabetes after transplantation for which she was taking insulin therapy; her other medications included aspirin, atorvastatin, prednisolone, ciclosporin, lansoprazole, amlodipine, bisoprolol, levothyroxine, quinine, pregabalin, sodium bicarbonate, alendronic acid, calcium carbonate, fentanyl and erythropoeitin. Her transplant function had been declining over the previous few months and an arteriovenous fistula had formed.

On examination, she was drowsy with a Glasgow Coma Score of 13/15. Her blood pressure was $140 / 100 \mathrm{mmHg}$ and she had evidence of significant fluid overload with an elevated jugular venous pressure and marked oedema of the face, lips and sacrum. She required $28 \%$ oxygen. Chest auscultation revealed reduced air entry bilaterally. A warm erythematous area was noted over the left shin. Blood glucose was high at 49.9 (normal range 3.5-7.8) $\mathrm{mmol} / \mathrm{L}$ with elevated blood ketones of $4.4(<0.6)$ $\mathrm{mmol} / \mathrm{L}$. Serum creatinine was 453 (normal range 55-125) $\mu \mathrm{mol} / \mathrm{L}$ (compared with $400 \mu \mathrm{mol} / \mathrm{L} 1$ month previously), urea 29.3 (normal range 1.7-7.1) mmol/L, potassium 4.5 (normal range 3.6-5.0) $\mathrm{mmol} / \mathrm{L}$ and sodium 128 (normal range 134145) $\mathrm{mmol} / \mathrm{L}$. Arterial blood gas analysis showed a $\mathrm{pH}$ of 7.1,

Authors: ${ }^{A}$ specialty registrar in nephrology and general internal medicine, Norfolk and Norwich University Hospital, Norwich, UK; ${ }^{B}$ consultant nephrologist, Norfolk and Norwich University Hospital, Norwich, UK standard bicarbonate 13.1 (normal range 22.0-30.0) $\mathrm{mmol} / \mathrm{L}$ and lactate 0.9 (normal range $0.7-2.1$ ) $\mathrm{mmol} / \mathrm{L}$.

Chest radiograph showed diffuse bilateral airspace opacification in keeping with pulmonary oedema or infective consolidation. A diagnosis of diabetic ketoacidosis (DKA) was made and she was started on a fixed rate intravenous insulin infusion in the emergency department. Intravenous fluids were not administered in view of her volume overload. She was given broad spectrum antibiotics for presumed sepsis secondary to either cellulitis or pneumonia. Her ketosis had resolved 6 hours later with a blood ketone level of $0.7 \mathrm{mmol} / \mathrm{L}$, but her hyperglycaemia persisted with blood glucose of $44 \mathrm{mmol} / \mathrm{L}$. She also had an ongoing metabolic acidosis with a venous $\mathrm{pH}$ of 7.1, venous bicarbonate $15.6 \mathrm{mmol} / \mathrm{L}$ and serum potassium $4.0 \mathrm{mmol} / \mathrm{L}$.

In view of her ongoing metabolic acidosis and concerns over fluid balance, she was transferred to the high dependency unit. Her intravenous insulin infusion was continued but without supplementary intravenous fluids. Over the next 12 hours, her blood glucose gradually fell to under $10 \mathrm{mmol} / \mathrm{L}$. Her acidosis also improved with an arterial $\mathrm{pH}$ of 7.31, standard bicarbonate $18 \mathrm{mmol} / \mathrm{L}$ and serum potassium $3.6 \mathrm{mmol} / \mathrm{L}$. Her urine output remained approximately $30 \mathrm{ml} /$ hour. Her dyspnoea, oxygen requirements, oedema and level of alertness improved significantly. Klebsiella pneumoniae was isolated from her blood cultures and appropriate antibiotics were continued. Her intravenous insulin infusion was discontinued after restarting an increased dose of basal insulin. The following day she was transferred to the renal ward where she was commenced on haemodialysis and she was discharged from hospital 1 week later on maintenance dialysis.

\section{Discussion}

The quandary in this patient's management was the conflicting demands of her DKA and renal failure. Established guidelines for the management of DKA recognise the vital importance of fluid resuscitation and potassium supplementation in most patients. However, their role in patients with advanced renal insufficiency (including those on dialysis), where they may be potentially dangerous, has not been well studied, despite the fact that diabetes mellitus is commonly present in patients with chronic kidney disease, either as the primary cause of their renal dysfunction or as a comorbidity. Literature on the topic is limited and includes three case reports ${ }^{1-3}$ and one review article. ${ }^{4}$ 
In patients with preserved renal function, osmotic diuresis is a key mechanism through which uncontrolled hyperglycaemia in DKA may cause harm, for example by causing profound hypovolemia and depletion of electrolytes such as potassium. ${ }^{5,6}$ This process can be significantly attenuated in patients with renal failure in whom oliguria or anuria results in a dramatic reduction in water and electrolyte losses. Hyperglycaemia in these patients may paradoxically lead to extracellular volume expansion with various proposed mechanisms, including increased thirst and water intake due to increased serum tonicity and osmotic shift of fluid from the intracellular to the extracellular compartment. ${ }^{4}$ Fluid administration to these patients can therefore be deleterious and needs to be considered carefully.

Similarly, while potassium supplementation (to prevent dangerous hypokalaemia) is usually vital in the management of DKA in patients with preserved renal function, ${ }^{5,6}$ potassium excretion is often impaired in patients with renal failure, and therefore routine administration of potassium to this group may lead to life-threatening hyperkalaemia. It has been reported that dialysis patients with hyperglycaemia have an increased frequency and severity of hyperkalaemia in comparison to patients with DKA and preserved renal function. ${ }^{7}$

A mainstay of DKA treatment is insulin; this improves both ketonaemia and hyperglycaemia. ${ }^{5,6}$ Correction of ketonaemia usually requires ongoing insulin infusion even after normalisation of blood glucose. Most protocols therefore recommend administration of a fixed rate intravenous insulin infusion and, when the blood glucose falls below $14 \mathrm{mmol} / \mathrm{L}$, concurrent administration of 5\% dextrose in order to prevent hypoglycaemia. As discussed above, this parallel fluid administration may be harmful in patients with renal failure and fluid overload. An alternative approach may be to monitor blood glucose levels and to administer aliquots of more concentrated dextrose solutions as required. In addition, insulin is renally-excreted and so doses may require modification in patients with renal impairment.

It has been reported that insulin treatment on its own can be helpful in resolving extracellular fluid expansion in chronic dialysis patients with severe hyperglycaemia. ${ }^{4}$ Interestingly, our patient had severe peripheral and pulmonary oedema on presentation which improved considerably following insulin treatment and resolution of her ketonaemia and hyperglycaemia. Her urine output was only moderate $(360 \mathrm{~mL}$ in 12 hours), so diuresis is unlikely to have been the main factor in this improvement.

\section{Conflicts of interest}

The authors have no conflicts of interest to declare.

\section{References}

1 Catalano C, Fabbian F, Di Landro D. Acute pulmonary oedema occurring in association with diabetic ketoacidosis in a diabetic patient with chronic renal failure. Nephrol Dial Transplant 1998;13:491-2.

2 Blicker J, Herd AM, Talbot J. Diabetic ketoacidosis in the dialysisdependent patient: two case- reports and recommendations for treatment. Can J Emerg Med 2004;6:281-4.

3 Gupta A, Rohrscheib M, Tzamaloukas AH. Extreme hyperglycaemia with ketoacidosis and hyperkalaemia in a patient on chronic haemodialysis. Haemodial Int 2008; 12 Suppl 2:S43-7.

4 Tzamaloukas AH, Ing TS, Siamopoulos KC et al. Body fluid abnormalities in severe hyperglycaemia in patients on chronic dialysis: review of published reports. J Diabet Complications 2008;22:29-37.

5 English P, Williams G. Hyperglycaemic crises and lactic acidosis in diabetes mellitus. Postgrad Med J 2004;80:253-61.

6 Savage MW, Dhatariya KK, Kilvert A et al. Joint British Diabetes Societies guideline for the management of diabetic ketoacidosis. Diabetic Med 2011;28:508-15.

7 Tzamaloukas AH, Ing TS, Elisaf MS et al. Abnormalities of serum potassium concentration in dialysis-associated hyperglycaemia and their correction with insulin: review of published reports. Int Urol Nephrol 2011;43:451-9.

Address for correspondence: Dr R Varma, Department of Renal Medicine, Norfolk and Norwich University Hospital, Norwich, Colney Lane, NR4 7UY, UK.

Email: ravi.varma@hotmail.co.uk 